\title{
Proposal on Financial Computing Algorithm and Analysis
}

\author{
Raghav Sethi \\ Student \\ Department Of Computer Science Engineering, \\ MPSTME, NMIMS University \\ Mumbai, India
}

\begin{abstract}
Algorithmic Finance is currently a nascent topic in today's time and is being researched around by various researchers around the globe. It involves the use of computer programs to buy and sell securities based on a pre-determined set of rules. Such algorithms have gained widespread use among institutional investors in major markets around the world. This paper discusses the various aspects of a financial algorithm and its prospects in the future. Algorithmic finance, being a relatively new field has plenty of scope to grow. The scope of this field of study, which we are inclined towards, is to develop software, which would help us understand the market trends. Since it is a computer algorithm, it would save a lot of time and effort, and also reduce the risk of our investment by a considerable margin. Algorithmic trading may be used in any investment strategy or trading strategy, including market making, inter-market spreading, arbitrage, or pure speculation (including trend following). The investment decision and implementation may be augmented at any stage with algorithmic support or may operate completely automatically.
\end{abstract}

\section{General Terms}

Trading, Stocks, Algorithmic trading, Financial instruments, Investment Strategy, Trading Strategy

\section{Keywords}

Financial instruments, Derivatives, Funds, Indices, Statistical graphs, small cap, mid cap, High frequency trading, algotrading

\section{INTRODUCTION}

Algorithmic finance, being a relatively new field has plenty of scope to grow. This software can change the way people trade. The reason being that it assists the investor at every possible step and also helps to reduce the risk by a good margin. This algorithm also helps the amateur investor to invest smartly and without the extra hassle of tons of background research. There are already pre existing algorithms such as the Monte Carlo trading algorithm and the genetic algorithm. These Algorithms have been reviewed in the second half of this research paper. The second half of this review paper not only discusses the currently existing algorithms, but also types of architectures proposed which would best suit the system. It also includes different softwares, which can be used to implement a new algorithm.

\section{EXISTING TECHNIQUES/SOFTWARES}

\subsection{Meta-Trader 5}

Meta Trader 5 offers a more powerful tool for developers of
Expert Advisors (trading robots) - the MQL5 IDE. It also includes a new object-oriented language, an editor with a debugger and a multicurrency strategy tester. Meta Trader 5 Trading Platform has an improved distributed architecture in which five servers balance the workload. This allows for scaling the platform and further enhancing its performance. Functionality, flexibility and security of the Meta Trader 5 Trading Platform are the key features of this product.

\subsection{Algo Trader}

Algo Trader is an all- in- one proprietary traders, quantitative hedge funds, and investment banks. It can trade Forex, futures, Options, Stocks \& Commodities on any market. It enables development, simulation and execution of multiple complex strategies in parallel. It is based on open source technologies like Hibernate, Spring, Esper, ActiveMQ, AndroMDA, Grails and QuickFIX/J.

Algo Trader follows following steps to deliver results to the customers :

1. Onsite and remote consultation and training

2. Automation and migration of existing strategies

3. Improving and optimizing existing strategies

4. Prototyping and back testing new strategies

5. Developing customized functionality

\subsubsection{Unique feature of Algo Trader}

\subsubsection{Incremental Innovation:}

1. It is a cost friendly method

2. Updates are made in small amounts

For example: Algo trader comes up with new type of formulas and algorithms so that the customer is shown a more accurate result of the amount of profit the customer is going to gain.

\subsubsection{Radical Innovation:}

1. Radical Invention on the other hand explores new technology.

2. It creates a new market rather than altering an existing one to fit its needs

3. It can be seen as a breakthrough innovation that is an innovation, which changes the market for a certain product completely.

\subsubsection{Evolution of Algo Trader}

After achieving great success with Microsoft, a renowned genius software developer retired as a multi-millionaire, then 
lost half of his retirement fortune in the stock market crash of 2000. That is when he realized a computer program could profit from the stock market much faster and better than any human ever could, including the most successful professional traders. So, in 2000 , he came out of retirement and spent the next 5 years writing over 5 million lines of code to create the world's first and only fully automated system for individual investors to generate profits in the stock market every day

\subsection{Monte Carlo Stimulation Method}

MC simulation is a technique that can help the customers estimate the risk and profitability of there trading strategy using the ATS. It can help to decide if the strategy is robust, what profit / drawdown can be expected from the strategy and if this trade strategy should be used at all. The basis of Monte Carlo method is running the same simulation a number of times, each time with small random changes. By simply reshuffling the trades, the final profit will stay the same, but drawdown can change a lot. Instead of drawdown $10 \%$ it might end with drawdown $30 \%$ just by changing the order of the trades.

\subsubsection{Processes Followed in Monte Carlo}

1. Flow Diagram

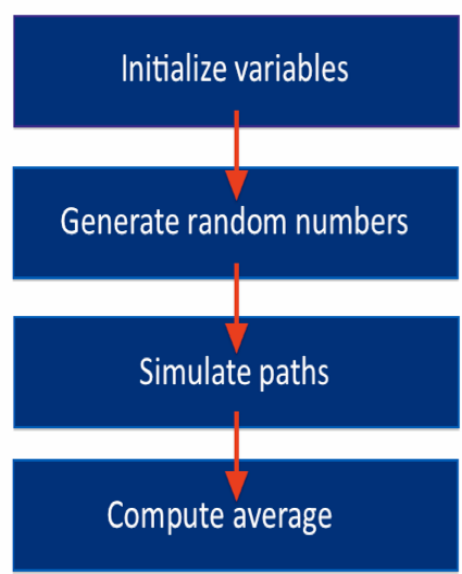

\section{Formula and Graph Used:}

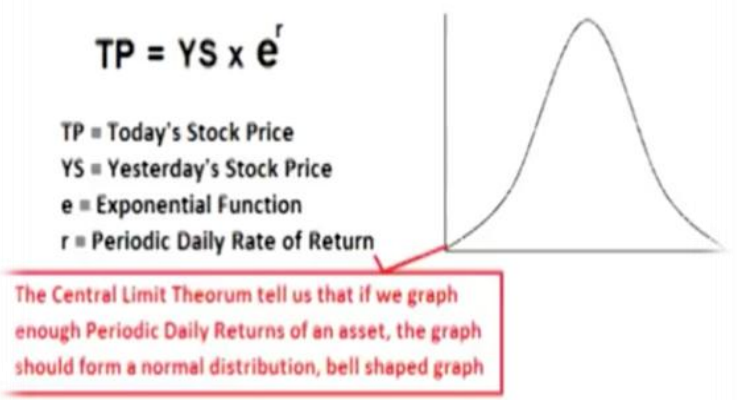

\section{Statistical Calculation}

Amount change in stock price $=$ the expected stock price + the effect of the volatility of people randomly buying and selling the stock over time has on that expected growth.

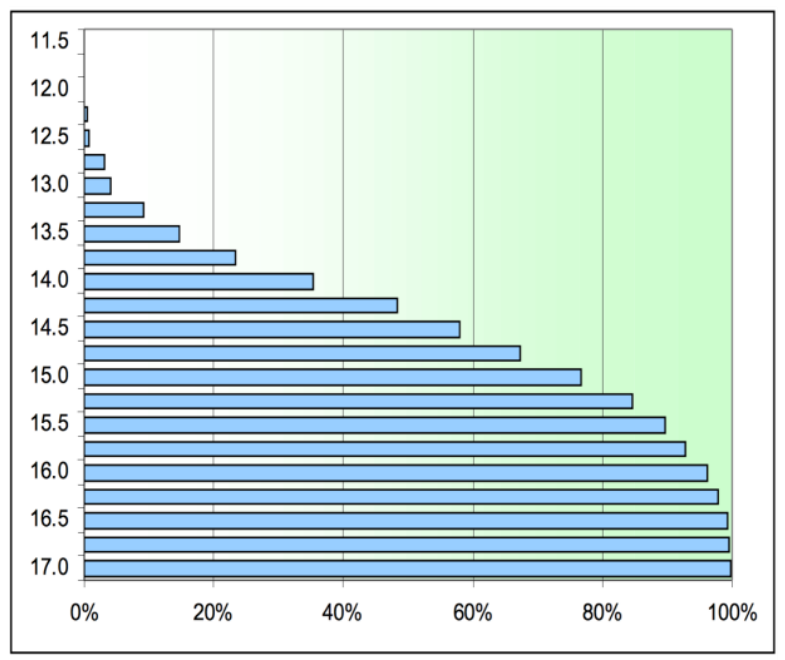

Figure 1: Probabilitv of Completion Within Specified Time (Months)

\subsubsection{Monte Caro vs. Algo Trader}

Monte Carlo Stimulation Method is better than Algo Trader. The answer lies in statistics, which are the basics of Monte Carlo. The customer can let a program run this reshuffling a hundred times and it will show what is the best, worst and average drawdown achieved during these random runs.

\subsection{Genetic Algorithm: Skipping Trades}

In this test some trades will be randomly missed (with given probability). In real trading often a trade can be missed because of platform or Internet failure, or simply because trading is paused for some time.

This test will give an idea how the equity curve might look like if some trades are randomly skipped.

\section{Formula Used}

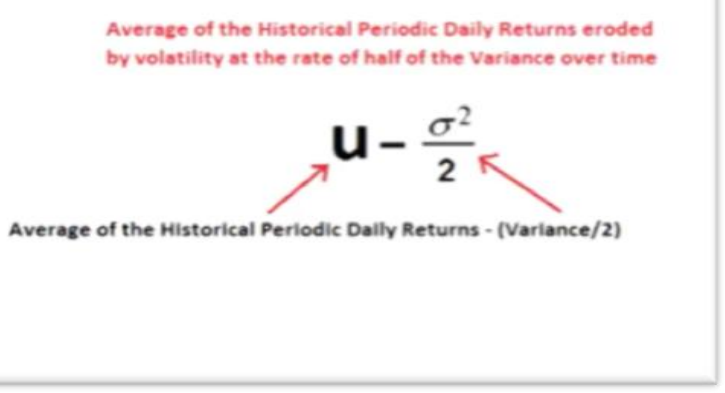

\section{Performance Statistics}

After performing a real time test on the the genetic algorithm following statistics were generated 
And here are some system performance statistics:

\begin{tabular}{|c|c|c|c|c|c|}
\hline Conf & Net Profit & $\operatorname{Max} 00 \%$ & Ret $D 0$ & REQP & $A R \%$ \\
\hline Orignal & $\$ 22018.71$ & & origin & $\mathrm{a} \mid 0.23 R$ & $14.88 \%$ \\
\hline $50 \%$ & $\$ 22018.7$ & $15,4 \%$ & 5.91 & $0.22 R$ & $14.88 \%$ \\
\hline $60 \%$ & $\$ 22018.7$ & $15.75 \%$ & 5.49 & $0.21 R$ & $14.88 \%$ \\
\hline $0 \%$ & $\$ 22018.69$ & $16.52 \%$ & 4.98 & $0.21 \mathrm{R}$ & $14.88 \%$ \\
\hline $80 \%$ & $\$ 22018.69$ & $18.36 \%$ & 4.4 & $0.2 R$ & $14.88 \%$ \\
\hline $90 \%$ & $\$ 22018.68$ & $21.22 \%$ & 3.87 & $0.18 R$ & $14.88 \%$ \\
\hline $91 \%$ & $\$ 22018.68$ & $22.76 \%$ & 3.53 & $0.18 R$ & $14.88 \%$ \\
\hline $92 \%$ & $\$ 22018.68$ & $23.07 \%$ & 3.41 & $0.18 R$ & $14.88 \%$ \\
\hline $93 \%$ & $\$ 22018.68$ & $23.12 \%$ & 3.26 & $0.18 R$ & $14.88 \%$ \\
\hline $94 \%$ & $\$ 22018.68$ & $23.24 \%$ & 3.24 & $0.18 R$ & $14.88 \%$ \\
\hline $95 \%$ & $\$ 22018.68$ & $23.59 \%$ & 3.16 & $0.18 R$ & $14.88 \%$ \\
\hline $96 \%$ & $\$ 22018.68$ & $23.6 \%$ & 2.94 & $0.17 R$ & $14.88 \%$ \\
\hline $97 \%$ & $\$ 22018.67$ & $23.99 \%$ & 2.68 & $0.17 R$ & $14.88 \%$ \\
\hline $98 \%$ & $\$ 22018.66$ & $24.28 \%$ & 2.64 & $0.17 R$ & $14.88 \%$ \\
\hline $99 \%$ & $\$ 22018.66$ & & 2.42 & $0.17 R$ & $14.88 \%$ \\
\hline $100 \%$ & $\$ 22018.65$ & & woest & case $R$ & $14.88 \%$ \\
\hline
\end{tabular}

The system that originally had drawdown around $16 \%$ now in worst case has drawdown $26 \%$, almost a double.

\subsection{Percentage Of Volume (POV)}

- The PoV (Percentage of Volume) algorithm addresses the problem of VWAP by using the actual traded volume of the day as benchmark. The idea is to have a constant percentage participation in the market along the trading period.

- If the quantity that remains to be traded is $\mathrm{Q}$, and the participation ratio is $\square$, the algo algo computes the volume $\mathrm{V}$ traded in the period $(\mathrm{t}-\Delta \mathrm{T}, \mathrm{t})$ and executes a quantity

\section{$\mathbf{q}=\min \left(\mathbf{Q}, \mathbf{V}^{*} \mathbf{g}\right)$}

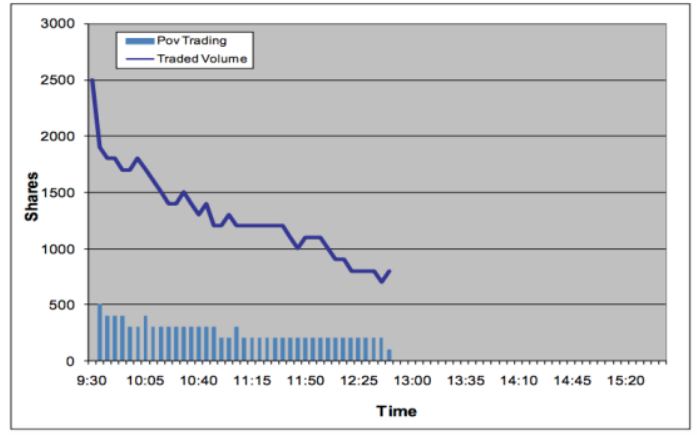

$\mathrm{V}(\mathrm{t})$ total volume traded in the market up to time $\mathrm{t}$ $\mathrm{Q}(\mathrm{t})$ number of shares that remain to be traded . (Q(0) initial quantity)

$$
\begin{aligned}
& Q(t+\delta t)-Q(t)=-\min [\gamma(V(t)-V(t-\delta t)), Q(t)] \\
& \begin{cases}\frac{d Q}{d t}=-\gamma \frac{d V}{d t} \quad ; Q(t)>\gamma \frac{d V}{d t} \delta t \approx 0 \\
\frac{d Q}{d t}=0 \quad ; Q(t) \leq \gamma \frac{d V}{d t} \delta t \quad \approx 0\end{cases} \\
& \frac{d Q}{d t}=-\gamma \frac{d V}{d t} \quad \therefore Q(T)-Q(0)=-\gamma \cdot V(T) \therefore \quad Q(0)=\gamma \cdot V(T) \\
& \frac{d Q}{d t} p(t)=-\gamma \frac{d V}{d t} p(t) \quad \therefore \int_{0}^{T}\left|\frac{d Q}{d t}\right| p(t)=\gamma \int_{0}^{T} \frac{d V}{d t} p(t) \\
& \frac{\int_{0}^{T}\left|\frac{d Q}{d t}\right| p(t)}{Q(0)}=\frac{\int_{0}^{T} \frac{d V}{d t} p(t)}{V(T)}
\end{aligned}
$$

\section{PROPOSED SYSTEM}

\subsection{Architecture}

The software architecture, which we believe would best suit the algorithm, which we want to implement, would be the Cyan Spring Architecture. Cyan Spring ATS can be run with a distributed architecture. Multiple servers can join a cluster to share the workload. Server can be a single server instance or multiple ones to form a server cluster. Client (CSTW) can connect to multiple servers in the same cluster and monitor all strategies run in the same cluster.

Cyan Spring ATS is built on top of the following software packages.They are all free and solid open source software.

- $\quad$ Apache Active MQ(Can be replaced by any JMS $)$

- Quick Fix/J

- $\quad$ Spring Frame Work

- $\quad$ Eclipse RCP

- Xstream

- $\quad$ SLF4J

- $\quad$ Apache LOG4J

- $\quad$ Apache Derby DB

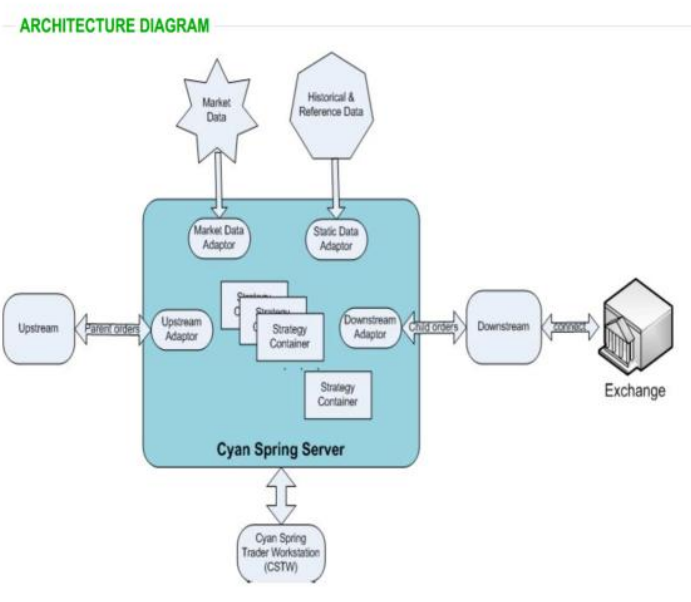

SYSTEM ARCHITECTURE

\subsection{Proposed Strategies}

A trading strategy is composed of two parts

- Strategy data- these are parameters for strategies $\square$

- $\quad$ Strategy logic- java codes that are built on top of the software (Cyan Spring ATS) framework.

\section{Single-order Strategies $\square$}

- A Single-order strategy, or single-order strategy, involves in trading only one instrument and one side transaction (buy or sell). It is usually created from an order, e.g. buy 0005.HK 200000 @ \$68.2.

The strategy is considered completed when the order is fully filled. The typical usage, though not limited to, is to avoid market impact while trading some large volume of orders.

\subsection{Strategy testing}

Executing all transactions in the database during simulation is 
useful for reporting but also incurs some additional processing time during. For trivial strategies that do not need to perform any sort of sophisticated querying based on transaction data an additional in-process / in-memory exchange simulator is available that uses Hash Maps as the underlying storage mechanism.

This will allow for significantly faster processing of transactions during simulation.

\section{Formulae Used}

1. Simulator simulator $=$ new Simulator();

2. Order order $=$ new

MarketOrder.Factory.newInstance(); order.setSecurity(security);

3. order.setStrategy(strategy);

4. order.setQuantity(qty);

5. order.setSide(Side.BUY);

6. simulator.sendOrder(order);

7. Position position $=$ simulator.findPositionByStrategyAndSecurity(strate gy, security);

During a simulation process the following steps are executed sequentially:

- The database is reset to its original state via the Reset Service .

- Esper Engines are initialized for the AlgoTrader Server as well as all strategies marked as autoActivate.

- All Esper Modules defined in column initModules and runModules of the table strategy are deployed .

- A Portfolio Rebalance is executed to distribute the initial CREDIT (of 1'000'000 USD) to strategies according to their allocation.

- For every Strategy Service the method initSimulation is invoked to execute any initialization tasks if necessary .

\subsection{Software Building Phase Prototype -}

\subsubsection{Technologies used}

- JavaJDKSE1.6

- Eclipse

\subsubsection{Exchange Simulator}

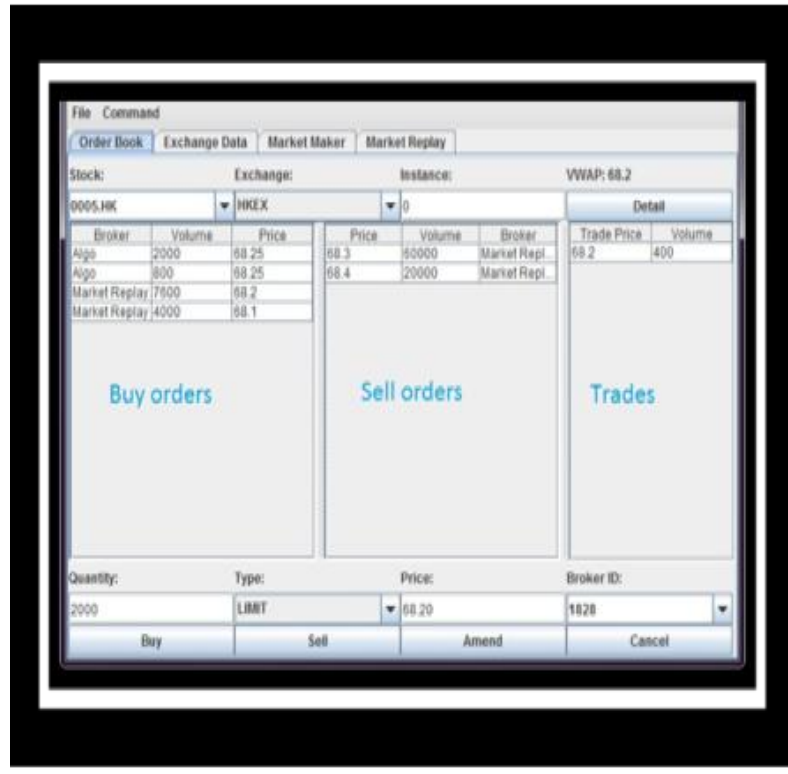

\subsubsection{Features}

- Pause order-the algo engine pauses the auto execution of order

- Stop order-the algo engine stops the auto execution of order

- Start order - the algo engine resume the auto execution of order

- Cancel order - the order is canceled. Algo engine withdraws orders and terminates the auto execution

- Show or hide filter - show or hide a filter panel where you may specify a filtering criteria to narrow down the parent orders shown in this view

- Enter order - entering a new order for algo execution.

\subsubsection{Statistical Analysis}

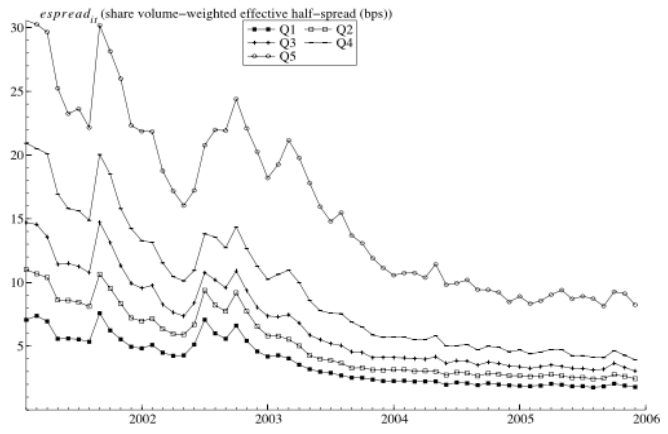

\subsubsection{CANDLE STICK GRAPH}

The proposed system uses the features of candle Stick graph.

Candle Stick graph is explained below:

- In order to create a candlestick chart, a data set is required that contains open, high, low and close values for each time period that is to be displayed.

- The hollow or filled portion of the candlestick is 
called "the body" (also referred to as "the real body"). The long thin lines above and below the body represent the high/low range and are called "shadows" (also referred to as "wicks" and "tails").

- The high is marked by the top of the upper shadow and the low by the bottom of the lower shadow.

- If the stock closes higher than its opening price, a hollow candlestick is drawn with the bottom of the body representing the opening price and the top of the body representing the closing price.

- If the stock closes lower than its opening price, a filled candlestick is drawn with the top of the body representing the opening price and the bottom of the body representing the closing price.

\section{Candlestick Formation}

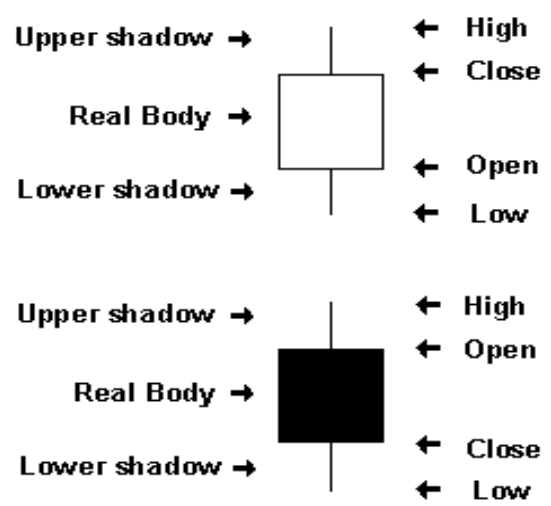

Compared to traditional bar charts, many traders consider candlestick charts more visually appealing and easier to interpret. Each candlestick provides an easy-to-decipher picture of price action. Immediately a trader can compare the relationship between the open and close as well as the high and low. The relationship between the open and close is considered vital information and forms the essence of candlesticks. Hollow candlesticks, where the close is greater than the open, indicate buying pressure. Filled candlesticks, where the close is less than the open, indicate selling pressure.

- Below is the Candle Stick used in the proposed algorithm for a time period of one hour

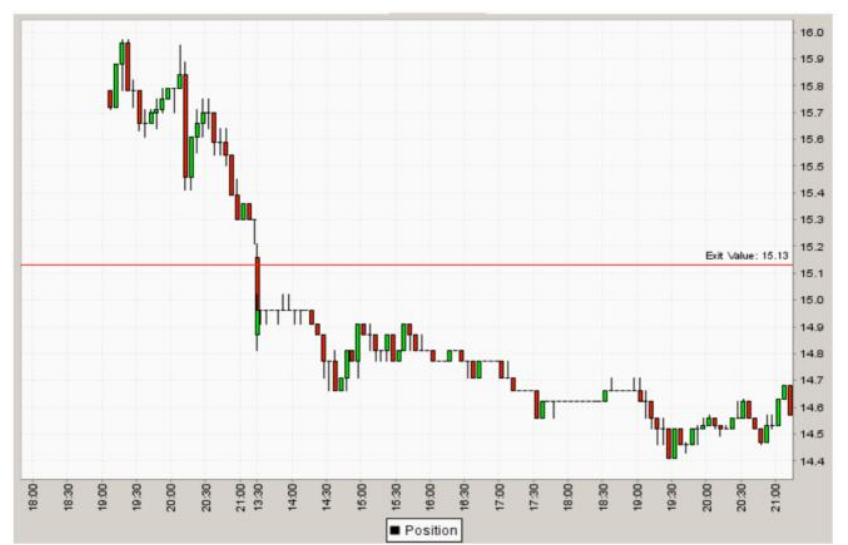

3. CONCLUSION
Thus the potential of algorithmic finance has been reviewed and the scope, which it has in the future, has ben determined. The existing algorithms have been analyzed and the flaws have been found out alongside the ways to overcome those faults. The potential architectures for our proposed system has been researched upon. The various simulators have also been looked into, which will help to implement our algorithm.

\section{FUTURE SCOPE}

Algorithms are widely recognized as one of the fastest moving bandwagons in the capital markets. Employing rulesbased strategies has enabled buy-side firms to increase productivity, lower commission costs and reduce implementation shortfall. Algorithmic trading cuts down transaction costs and allows investment managers to take control of their own trading processes. By breaking large orders into smaller chunks, buy-side institutions are able to dis- guise their orders and participate in a stock's trading volume across an entire day or for a few hours. More sophisticated algorithms allow buy-side firms to fine-tune the trading parameters in terms of start time, end time, and aggressiveness. In today's hyper-competitive, cost-conscious trading environment, being the first to innovate can give a broker a significant advantage over the competition both in capturing the order flow of early adopters and building a reputation as a thought leader. In the future, a range of important drivers of change, acting alone and in concert, will substantially influence the development and uptake of computer-based trading.

\section{REFERENCES}

[1] Adaptive arrival place by Robert Almgren and Julian Lorenz

[2] Monte-Carlo Evaluation of trading systems by Timothy Masters

[3] Algorithmic Trading and Computational Finance by Michael Kearns (Computer and information science, University of Pennsylvania)

[4] Online Financial Algorithms by Sandeep Kumar and Deepak Telang (Thapar University, Patiala)

[5] Daniel Safarik Algorithms a la Carte. Wall Street \& Technology, January 30, 2006.

[6] Nenad Yashruti Seeing Is Believing. Head Trader, Freestone Capital Management; Wall Street \& Technology; November 21, 2006.

[7] John Bates Algorithmic gymnastics - keeping at least one vault ahead of the rest. Hedge Funds Review, June 2006

[8] Katherine Heires Algorithms and Clearing Wrapped Up in One Algorithmic Trading. Wall Street \& Technology; May 25, 2006

[9] Daniel Safarik The 'Holy Grail': Pre-Trade Analytics. Wall Street \& Technology March 01, 2005.

[10] I. Domowitz, H. Yegerman, "Measuring and interpreting the performance of broker algorithms" in ITG Inc. Research Report, August 2005. 